\title{
Validación de un cuestionario para evaluar la calidad asistencial en Podología
}

\section{Validation of a questionnaire to assess the quality of care in Podiatry}

\author{
Manuel Coheña Jiménez ${ }^{1}$, José Ramos Galván ${ }^{2}$, Ramón Mahillo Durán ${ }^{3}$ \\ ${ }^{1}$ Doctor en Podología. Asistente Honorario Departamento de Podología. Facultad de Enfermería, Fisioterapia y \\ Podología. Universidad de Sevilla, España.cohmanu@yahoo.es \\ ${ }^{2}$ Doctor en Podología. Profesor Titular. Departamento de Podología. \\ Facultad de Enfermería, Fisioterapia y Podología. Universidad de Sevilla, España.jramos@us.es \\ ${ }^{3}$ Doctor en Podología. Subdirector del Área Clínica de Podología. Facultad de Enfermería, Fisioterapia y Podología. \\ Universidad de Sevilla, España. rmahillo@us.es
}

Correspondencia:

Manuel Coheña Jiménez

Departamento de Podología

Centro Docente de Fisioterapia y Podología

c/ Avicena s/n

E-41009 Sevilla

Correo electrónico: cohmanu@yahoo.es

Fecha de recepción: 8 de febrero de 2013

Fecha de aceptación: 22 de abril de 2013

Los autores declaran no tener ningún tipo de interés económico o comercial.

\section{RESUMEN}

Objetivos: En este trabajo se elabora, pilota y valida un cuestionario para evaluar la percepción de calidad asistencial en el ámbito específico de la Podología.

Métodos: Se elaboró un cuestionario sobre calidad asistencial percibida en Podología, con 27 ítems agrupados en tres áreas: calidad asistencial, criterios de referencia e información complementaria socio-demográfica, partiendo de los modelos teóricos de calidad de servicio. Las dimensiones que se exploran son accesibilidad, cortesía, comunicación, capacidad de respuesta, seguridad y tangibilidad. Se realizó un pilotaje de 30 cuestionarios, con posterior análisis de propiedades psicométricas de validez y fiabilidad mediante análisis factorial de componentes principales y cálculo de alpha de Cronbach.

Resultados: La prueba de adecuación de Kaiser-Meyer Olkin fue de 0,852 y una prueba de esfericidad de Barlett de 1194,158. La varianza acumulada de 63,804\%. El alpha de Cronbach fue de 0,910. Se clasificaron en 6 factores compuestos por varios ítems, denominados Aspectos clínicos, Información, Administración e instalaciones, Accesos, Tiempos y espera.

Conclusiones: Presentamos un cuestionario que tiene garantías de validez y fiabilidad para evaluar la percepción de calidad asistencial en el ámbito específico de la Podología. El cuestionario diseñado es una herramienta útil para el desarrollo de estudios posteriores y de aplicación en contextos lo más parecidos posibles al nuestro que incluye el resto de clínicas universitarias podológicas. Su aplicación nos permitirá conocer debilidades y fortalezas relacionadas con la calidad asistencial percibida en Podología.

Palabras clave: cuestionario; calidad asistencial; percepción; podología; validación.

\section{ABSTRACT}

Objectives: This study constructs, pilot and validate a questionnaire to evaluate perception's quality of care in the specific context of Podiatry.

Methods: We prepared a questionnaire on quality of care received in Podiatry, with 27 items grouped into three areas: quality of care, benchmarks and socio-demographic information, based on theoretical models of service quality. The dimensions that are explored are accessibility, courtesy, communication, responsiveness, security and tangibility. A pilot study of 30 questionnaires was carried out, with subsequent analyses of psychometric properties of validity and reliability using principal components factor analysis and calculation of Cronbach's alpha.

Results: The adequacy test Kaiser-Meyer Olkin was 0,852 and Bartlett's test of sphericity was of 1194.158. An 
cumulative variance of $63,804 \%$. Cronbach's alpha was 0,910 . Ranking in six factors consisting of several items: Clinical Aspects, Information, and facilities management, access and waiting times.

Conclusions: We present a questionnaire that has guarantees of validity and reliability to assess the perception of quality of care in the specific context of Podiatry. The designed questionnaire is a useful tool for developing and implementing further studies in similar contexts as possible to ours including other podiatric university clinics. Its implementation will allow us to know strengths and weaknesses related to the quality of Care received in Podiatry.

Key words: questionnaire; quality of care; perception; podiatry; validation.

Sumario: 1. Introducción. 2. Material y métodos. 3. Análisis estadístico. 4. Resultados. 5. Discusión. Agradecimientos. Bibliografía.

Referencia normalizada: Coheña Jiménez, M., Ramos Galván, J., Mahillo Durán, R. Validación de un cuestionario para evaluar la calidad asistencial en Podología. Rev. Int. Cienc. Podol. 2013; 7(2): 99-108. 


\section{INTRODUCCIÓN}

Históricamente el concepto de calidad asistencial ha estado presente en los servicios sanitarios, siendo un pilar básico de éstos, un reto y desafío de los sistemas de salud del siglo XXI. Regulados por el derecho a la protección de la salud, Ley General de Sanidady Ley de Cohesión y Calidad sanitaria. La preocupación de la calidad asistencial por los podólogos está presente en nuestro Código Deontológico.

El Área Clínica de Podológica (ACP) de la Universidad de Sevilla es un centro docenteasistencial, referente nacional, que atiende la salud podológica. Las características de sus usuarios requieren estudiar la calidad asistencial percibida, mediante el diseño de un cuestionario específico desde la perspectiva del paciente, centrándolo como eje del sistema ${ }^{1}$. La calidad sólo se mejora cuando los procesos están diseñados con la premisa de satisfacer las necesidades de los usuarios ${ }^{2,3,4}$. No existen trabajos científicos similares de asistencia podológica recibida en clínicas universitarias, aunque si son numerosos en otros contextos: consultas externas hospitalarias ${ }^{5}$ y Atención Primaria ${ }^{6}$.

Fundamentalmente existen dos modelos de calidad de servicio ${ }^{7}$, con diferentes conceptualizaciones y formas de entenderla: Grönroos en 1.982 y Parasuraman, Zeithalm y Berry en 1.986. La introducción del concepto de percepción en la definición de calidad sitúa al usuario como el único que puede determinar si un servicio es o no de calidad ${ }^{2}$ y la satisfacción percibida como elemento fundamental y como indicador válido de la calidad de los cuidados sanitarios prestados ${ }^{8}$.

En España, existen estudios de satisfacción y de calidad percibida, desde una mirada del usuario, que han utilizado el Servqual como soporte teórico y como base instrumental ${ }^{9,10}$. El Servperf considera que la calidad de un servicio debe ser medida tan sólo a través de las percepciones, sin necesidad de compararlas con las expectativas ${ }^{11}$. Es necesario conocer la opinión de los usuarios para incrementar la calidad asistencial y mejorar los tratamientos que aplicamos en Salud ${ }^{12}$.

La gestión sanitaria debe abandonar la idea de que es posible construir una calidad de ser- vicio objetiva, para poder avanzar en construir estrategias efectivas de comunicación y consenso de criterios comunes de distinción de calidad de servicio, para lograr efectiva satisfacción y lealtad de los pacientes ${ }^{13}$.

Este trabajo surge de la inexistencia de trabajos similares en Podología, y tiene por objeto elaborar, pilotar y validar un cuestionario para evaluar la percepción de calidad asistencial en el ámbito específico de la Podología.

\section{MATERIAL Y MÉTODOS}

El cuestionario fue elaborado mediante una adaptación de los modelos teóricos de Sevqual y Servperf, entre otros. En una primera fase, se realizaron la formulación de los ítems totales del cuestionario con semejanzas con los modelos originales, adaptándolos al contexto especifico de Podología. Posteriormente, se realizaron cambios en la formulación, comprensibilidad e incluso la supresión de ítems para evitar un cuestionario tedioso. Por consenso, quedaron agrupados en diferentes dimensiones que fueron acordadas tras varias sesiones de trabajo entre diversos profesores del Departamento de Podología y un grupo de doctores y gestores de la Universidad de Sevilla. Se pretendieron explorar: accesibilidad, cortesía, comunicación, capacidad de respuesta, seguridad y tangibilidad.

El cuestionario resultante se componía de los siguientes apartados: uno principal de calidad asistencial, uno central con criterios de referencia y uno final con información complementaria sobre datos socio-demográficos: sexo, edad, nivel de estudios, profesión actual y localidad de procedencia.

Los datos de calidad asistencial:

1. La facilidad de los trámites que tuvo que hacer en la ventanilla de Admisión.

2. La facilidad de los trámites que ha tenido que hacer para volver a pedir cita.

3. La facilidad de acceso para llegar al centro (horario, aparcamiento, transporte público).

4. Las adaptaciones para discapacitados en el acceso al centro.

5. El tiempo transcurrido desde que pidió la cita hasta la consulta. 
6. El tiempo de espera para ser atendido.

7. El horario de las citas.

8. El trato recibido por parte del personal.

9. La amabilidad y cortesía del personal que le ha atendido.

10. El nivel de seguimiento personalizado de su caso.

11. El nivel de satisfacción de los servicios ofrecidos en el centro en relación con los precios.

12. La disponibilidad de información escrita (folletos, dípticos) en el centro.

13. La información sobre su problema de salud que el personal del centro le ha proporcionado.

14. La información sobre su proceso de salud facilitada a sus familiares y/o acompañantes.

15. La claridad con que le explicaron el tratamiento y pautas.

16. El interés del personal por solucionar su problema de salud.

17. La confianza y seguridad que el personal que le atiende y le ha transmitido.

18. El cuidado de su intimidad durante la consulta y exploración.

19. Su opinión sobre el tratamiento propuesto.

20. El estado físico del centro (sala de exploración, gabinetes).

21. La comodidad de la sala de espera.

22. La Tecnología de los equipos (modernos y suficientes) para el diagnóstico y tratamiento.

23. Las indicaciones (señalizaciones) para orientarse y saber dónde ir en el centro.

24. ¿Sabría identificar a las personas que le han atendido, profesor, estudiante, personal auxiliar, etc.?

Los datos de criterios de referencia:

25. Indique su nivel de satisfacción global con la atención recibida en el centro.

26. Recomendaría este centro a otras personas.

27. Conoce el nombre del Profesor/estudiante que le atendía.

Para las respuestas se utilizó una escala Likert de 0 a 5 puntos para la mayoría de ítems (25), que oscilaba de "muy mala" (1) a "muy buena" (5) en los 24 ítem. El ítem 25 de "nada satisfecho" a "muy satisfecho". El ítem 26 contenía tres posibles respuestas que oscilaba de "Sin dudarlo" a "No lo recomendaría" y el ítem
27 una respuesta dicotómica $\mathrm{Si} / \mathrm{No}$. Se realizó un pilotaje de 30 cuestionarios para mejorar la efectividad ${ }^{14}$. La participación se realizó previo consentimiento informado de los usuarios del centro.

Este proyecto contó con la autorización del Comité ético de experimentación del Vice-rectorado de Investigación de la Universidad de Sevilla.

\section{ANÁLISIS ESTADÍSTICO}

Se realizó un análisis descriptivo de los datos socio-demográficos y del cuestionario específico de calidad asistencial percibida. Primero, se valoró la correlación ítem-escala. La correlación realmente elevada se muestra entre la puntuación general del cuestionario y el ítem 25 con un índice de correlación de Pearson de 0,553 . La mayor aportación a la varianza total fue del ítem 25.

Las propiedades psicométricas del cuestionario comenzaron con el análisis de la dimensionalidad. Mediante un análisis factorial exploratorio sobre componentes principales, con posterior rotación varimax de los componentes para conocer la estructura de los factores. La adecuación del análisis se comprobó mediante la medida de Kaiser-Meyer Olkin (KMO) y la prueba de esfericidad de Barlett. La consistencia interna de los ítems de cada uno de los factores resultantes se exploró a partir del coeficiente de alpha de Cronbach (los superiores a 0,8 son indicativos de fiabilidad). Todos los cálculos se realizaron con el paquete estadístico SPSS 16.0 para Windows.

\section{RESULTADOS}

De los 379 cuestionarios entregados un 63,59\% fue respondido por mujeres. La edad media de 53,23 años. Según nivel de estudios la mayoría (60\% estudios primarios) y la mayoría, $62,8 \%$, de la ciudad de Sevilla.

La matriz de correlaciones (Tabla 1) mostró dos ítems (3 y 24) muy independientes, se correlacionan muy poco con el resto del cuestionario. Posteriormente y previo al análisis factorial, se valoró la correlación ítems-escala 
y se eliminó el ítem 4 debido al alto porcentaje de respuestas en blanco que ofreció durante el pilotaje.

En la Figura 1 observamos que el ítem 25 obtiene la mayor parte de la varianza explicada. El ítem 26 se correlaciona significativamente con el ítem 25 pero menos con la puntuación total de cuestionario. La correlación entre el ítem 26 y la puntuación total es de $\mathrm{r}=0,446$, con lo que parte de esta correlación ya está explicada con la variable "satisfacción global" y lo que aportó fue una porción mínima.

Se realizó el análisis factorial a los 23 ítems restantes, con unos valores de la prueba de adecuación de KMO de 0,852 y una prueba de esfericidad de Barlett de 1194,158. Mediante el análisis factorial (Tabla 2) exploratorio de componentes principales se identificaron cinco

Tabla 1. Matríz de correlaciones

\begin{tabular}{|c|c|c|c|c|c|c|c|c|c|c|c|c|c|c|c|c|c|c|c|c|c|c|c|}
\hline \multicolumn{24}{|c|}{$m$} \\
\hline ift. & & 2 & 3 & 5 & 6 & 7 & 8 & 9 & 10 & 11 & 12 & 13 & 14 & 15 & 6 & 7 & 8 & 19 & 20 & 21 & 22 & 23 & 24 \\
\hline \multirow[t]{2}{*}{1} & & & $\overline{39}$ & 7 & $\overline{51}$ & $\overline{54}$ & & $\overline{33}$ & 325 &, 421 & 287 & $\overline{62}$ &, 413 & 189 & 69 & 85 & 301 & 232 &, 401 & 364 & 307 & 370 &, 093 \\
\hline & & 0 & 04 & 0 & 0 & 00 & 00 & 001 &, 001 & , 000 & ,004 &, 000 & ,000 & ,064 & 000 & 000 & 003 & 022 &, 000 & 000 & 002 & 00 & 365 \\
\hline \multirow[t]{2}{*}{2} & & &, 320 & 360 & 86 & 98 & 401 & 356 & 185 &, 322 & 339 & 469 &, 412 & 202 & ,47 & 456 & , 260, &, 308 & 376 & 420 & 261 & 298 &, 01 \\
\hline & 000 & & ,001, & ,000, & ,004 & ,003, & ,000 & ,000 & ,070 & , 001 & ,001 & ,000 & ,000 & ,047, & ,000 &, 00 & 010 & 00 & 00 & 000 & 0 & 003 & 90 \\
\hline \multirow[t]{2}{*}{3} & 89 & 320 & & ,134 & , 191 &, 158 &, 074 &, 078 &, 213 & ,253 &, 134 & .031 & $\begin{array}{l}, 076 \\
\end{array}$ &,- 075 & , 09 &, 082 & 249 &, 169 & .084 & 206 & 095 & 045 &,- 10 \\
\hline & 004 & ,001 & & 189 & , 060 & , 122 & ,471 & ,445 &, 036 & , 012 & 190 & ,765 & ,461 & ,463 &, 378 & ,427 & ,014 & ,099 &, 412 & ,043 & 356 & 660 & ,324 \\
\hline \multirow[t]{2}{*}{5} &, 473 & 360 &, 134 & &, 399 & ,407 & 2,254 &, 352 & 255 & ,395 &, 261 & 252 & 256 & 245 & ,379 & 400 & 290 &, 353 &, 353 &, 317 & 423 & 240 & , 168 \\
\hline &, 000 & ,000 & , 189 & & ,000 & , 000 &, 012 & ,000 &, 012 & , 000 & , 010 & ,013 & , 011 & , 015 & ,000 & 000 & ,004 & , 000 & ,000 &, 002 & ,000 & , 018 & , 100 \\
\hline \multirow[t]{2}{*}{6} & 351 & 286 & ,191 &, 399 & & 440 & 283 & 186 &, 115 & 218 & 301 & 384 & 377 &, 219 & 257 &, $32=$ &, 431 & 405 & 287 &, 319 &, 312 & .12 &, 113 \\
\hline & , 000 & ,004 & , 660 & ,000 & & ,000 & ,005 & 068 & 260 & .032 & .003 & ,000 & , 000 &, 031 & 011 & ,001 & ,000 & ,000 & .004 &, 001 & ,002 & 243 & ,271 \\
\hline \multirow[t]{2}{*}{7} &, 404 & 298 & 158 &, 407 & $\overline{40}$ & &, 262 &, 309 &, 224 &, 410 & 280 &, 331 &, 361 & 263 &, 323 & 398 &, 369 & 27 & 295 & 259 &, 196 & 328 &, 206 \\
\hline & ,000 & ,003 & ,122 & ,000 & 000, & & ,009 & ,002 & 028 &, 000 & ,005 &, 001 &, 000 & ,009 & ,001 & , 00 & 000 &, $0 \mathrm{c}$ & ,003 & , 011, & 062 & ,001, & ,043 \\
\hline \multirow[t]{2}{*}{8} &, 390 &, 401 & 74 & 4 & ,283 & 62 & &, 600 & 377 & 356 & 237 &, 315 & 92 & 17 & 78 & 52 & , 217 & &, 354 & 305 &, 195 & 33 &, 108 \\
\hline &, 000 & ,000 & ,471 & , 012 & ,005, & 09 & & ,000 & ,000 & , 000 & 019 &, 002 & 000 &, 002 & , 000 &, 00 & ,033 & ,00 & 000 & ,002 & ,055 & ,001 & ,292 \\
\hline \multirow[t]{2}{*}{9} &, 333 &, 356 & 78 &, 3 &, 186 &, 3 & $\overline{00}$ & & ,322 & ,354 & 282 & 34 & 363 & & 369 & $\overline{42}$ & 283 & 19 &, 436 &, 196 &, 276 & 280 & 237 \\
\hline & ,001 & ,000 & ,445 & ,000 & ,068 & ,002 & ,000 & &, 001 & 000 &, 005 &, 002 &, 000 &, 003 & ,000 &, $0 c$ &, 005 & ,061 &, 000 & .055 & , 006 & 00 & , 019 \\
\hline \multirow[t]{2}{*}{10} &, 325 &, 185 &, 213 &, 255 & 115, & ,224 &, 377 & 22 & & 485 & 296 & $\overline{324}$ & 278 &, 389 & ,611 &, 59 & 339 & 47 & 226 & ,244 &, 413 & 384 &, 235 \\
\hline & ,001 & 070 & ,036 & , 012 & ,260 & ,028, & ,000 & ,001 & & 000 &, 003 & ,001 & 006 & ,000 &, 000 &, 00 & ,001 & 0 & ,026 &, 016 & ,00 &, 000 &, 021 \\
\hline \multirow[t]{2}{*}{11} &, 421 &, 322 &, 253 & ,395 &, 21 &, 41 &, 356 &, 354 & 485 & & 285 &, 410 & 409 &, 36 &, 52 &, 5 & 375 &, 3 &, 402 &, 369 &, 48 &, 44 &, 205 \\
\hline & , 000 & ,001 & , 012 & ,000 & ,032 & , 000 &, 000 & ,000 & , 000 & & ,005 & ,000 & 000 & , 000 & ,000 &, $0 c$ & ,000 & ,00 & ,000, & ,000, & ,000 & , 000 & ,044 \\
\hline \multirow[t]{2}{*}{12} &, 287 & 339 & ,134 & 261 & 301 & 280 &, 237 &, 282 & 296 & 285 & & 570 & 402 & ,477 & 362 & 43 & 265 & 25 & ,192 & 054 &, 231 &, 25 &, 191 \\
\hline & ,004 & ,001 & 190, & ,010 & ,003 & , 005 & ,019 & ,005 & ,003 & ,005 & & ,000 & ,000 & ,000 & ,000 &, $0 c$ & , 009 & , 012 & ,060 &, 597 & 02 & ,01 &, 061 \\
\hline \multirow[t]{2}{*}{13} &, 362 & 469 & 31 &, 252 & 38 & 33 &, 315 &, 314 & 324 & , 410 &, 570 & &, 733 &, 75 &, 54 & .6 &, 44 &, 4 & 27 & 24 & , 49 & 4 & ,331 \\
\hline & ,000 & ,000 & 5 &, 013 & , 000 & 1 & ,002 & 02 & ,001 & ,000 & ,000 & &, 000 &, 000 & ,00 & 0 & ,00 & & ,006 & 01 & 000 & 000 & 001 \\
\hline 14 & ,413 &, 4 & & & &, 3 & 2 & 53 &, 278 &, 4 & 02 & $3=$ & & &, 493 & & & &, 302 & 383 & 319, & 32 & 271 \\
\hline &, 000 & 000, & 46 & 11 & ,000, & 000 & ,000 & ,000 & ,006 & ,000 & 000 & ,000 & & 000 & 00 & 0 & , & , & , 00 & 00 & , 00 & , oc & ,007 \\
\hline 15 & , 189 & 202 &,- 075 & 245 & 219 & 26 &, 317 &, 301 & 389 & 366 & ,477 & 757 & ,672 & &, 53 & 60 & ,27 &, 47 & ,22 & 05 & 33 & 404 & 307 \\
\hline & ,064 & ,047 & ,463 & ,015 & ,03 &, 006 & ,002 & ,003 & ,000 & ,000 & ,000 & ,000 & , 000 & & , 00 & 0 & 00 & , & ,028 & 607 & ,001 & 00 & ,002 \\
\hline$\sqrt{16}$ &, 469 &, 471 & ,091 &, 379 &, 257 &, 323 &, 478 & 369 & ,611 &, 526 &, 362 & 544 &, 493 & , 53 & & 7,7 &, 45 &, 5 & .52 & 45 & 50 &, 50 &, 269 \\
\hline &, 000 & ,000 & ,378 & ,000 & &, 00 & ,000 & ,000 & ,000 & , 000 & ,000 & ,000 & ,000 & ,000 & &, 00 & ,000 & 0 & 000 & , 00 & ,00 & ,00 & ,008, \\
\hline$\overline{17}$ &, 485 & 4,456 &, 082 &, 400 & , 323, & 398 &, 522 &, 422 &, 595 &, 543 & ,439 & ,616 & ,636 & ,606 & ,794, & & ,447 & .56 &, 446 &, 45 &, 53 &, 53 & 266 \\
\hline &, 000 & ,000 & ,427 & , 000 & 001 & ,000 & ,000 & ,000 & ,000 & ,000 & 000 & 000 & , 000 & ,000 & 000 & & ,000 &, $0 c$ & ,000 & 00 & , 00 & 00 & ,009 \\
\hline 18 &, 301 & 260 & 249 &, 29 &, 4 &, 369 &, 217 & 283 & ,339 & ,375 & 265 & ,447 & ,395 & 278 &, 451 & 4 & & 6 & , 36 &, 45 & 58 & 39 &, 370 \\
\hline &, 003 & , 010 &, 014 & , & ,000 & & ,033 & ,005 & ,001 &, 000 & ,009 & ,000 & ,000 & ,006 & 000 & 0 & & & , 00, & ,000 & , & , & , 000, \\
\hline 19 &, 232 & 308 & 169 &, 35 &, 4 & 27 & 33 & 191 & ,473 & 328 & 255 & ,486 &, 446 & ,475 &, 54 &, 50 & 0 & &, 35 &, 42 & ,5 &, 4 & ,387 \\
\hline &, 022 & ,002 & 99 & 000, & ,000, & , 006 & 001 & 061 & ס000 & 001 & , 012 & ,000 & ,000, & ,000 & 000 &, 00 & , 000 & & , &, 000 &, 000 &, 000 &, 000 \\
\hline 20 &, 401 & 376 & ,084, &, 35 & , 28 & ,295 &, 354 & 436 & 2226 & ,402 & ,192 & 279 & ,302 &, 223 &, 526 &, 44 &, 36 & & & 496 & ,488 & ,467 & 210 \\
\hline & , 000 & ,000 & ,4 & , & 4 & & 0 & 100 & ,026 & ,000 & ,060 & ,006 & ,003, & 28 & 00 & ,000, & , 000 &, 00 & & b00 & 300 & 000 & ,039 \\
\hline 21 &, 364 &, 420 & 206 & .317 & 19 & 59 &, 305 & 196 & 244 & 369 & 054 & 247 & 383 &, 053 & 459 & .45 & 51 & 4,4 & ,496 & & 4866 &, 356 &, 223 \\
\hline &, 000 & ,000 & ,043 & ,002 & ,001 & , & ,002 & 055 &, 016 & 000 & ,597 & ,015 & , 000 & 607 & ,000 & ,000 & ,000 & ,000 & 00 & & ,000, & , 000, & ,028 \\
\hline 2 & ,307 & 261 & ,095 & .42 & 3 & 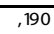 & 1955, & ,276 &, 413 & 480 & ,231 & ,490 &, 319 &, 332 &, 50 &, 5 &, 5 &, 5 & 48 & & &, 40 & 29 \\
\hline &, 002 & , 010 &, 35 & , & 0 & 06 & 0 & ,006 & , 000 & ,000 & 023 & ,000 &, 00 & 0 & 0 &, 00 & 0 & , & 000 & & & 00 & , 00 \\
\hline 23 & ,370 & 298 & ,045 & 240 &, 12 & ,328 &, 333 & 280 & 384 & 447 & 250 & .419 & 329 & ,404 &, 509 &, 530 &, 395 & & , 67 & 356 & & & 454 \\
\hline & ,000 & ,003 & 660 & ,018 & 43 & & ,001 & ,005 & ,000 & ,000 & , 013 & 000 & ,001 & ,000 & ,000 & , & ,000, & ,000 & |000 & 000 & 000 & & 00 \\
\hline &, 093 &,- 013 & -101 & 16 &, 1 & , &, 108 &, 237 & 235 & 205 & 191 &, 331 & 271 & 307 & ,269 & 26 & 370 & , &, 210 & 223 & 299 &, 454 & \\
\hline & & -5 & 324 & , 10 & & & 292 & 0 & ,021 & ,044 & ,061 & ,00 & , 00 & & 008 & 00 & | & , & & ,028, & ,003 & , 000 & \\
\hline
\end{tabular}




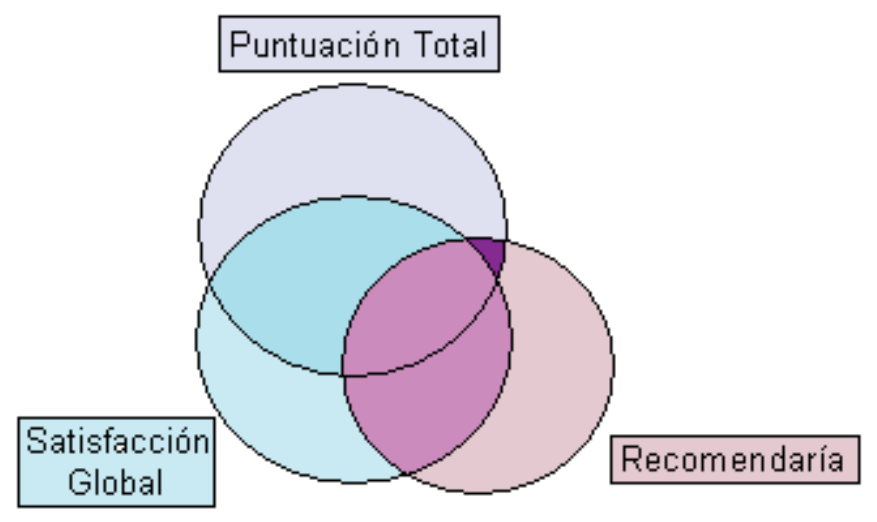

Figura 1. Diagrama de Puntuación total- Satisfacción global- Recomendaría.

componentes con una varianza acumulada del $63,804 \%$, que son los factores con valor superior a 1. Los valores de consistencia de alpha de Cronbach fueron de 0,91.

Intentamos aumentar la fiabilidad mediante la eliminación de los ítems 3 y 24 que guardaban menos relación con el cuestionario. Estudiamos el índice de consistencia interna tras su eliminación con resultado poco significativos (ítem 3 el índice del alpha de Cronbach pasaba de 0,910 a 0,919 y el del ítem 24 a 0,912). Posteriormente se procedió a la rotación ortogonal de la matriz factorial (tabla 3 ) y la clasificación de los factores (Tabla 4).

\section{DISCUSIÓN}

Se ha elaborado un cuestionario con cinco dimensiones que nos permite conocer la percepción de calidad asistencial de los usuarios del ACP de la Universidad de Sevilla. La principal fortaleza reside en el modelo teórico ele- gido para la elaboración del cuestionario y la necesidad de adaptar los ítems al contexto de aplicación, mejorando su validez predictiva o de contenido ${ }^{15}$.

El resultado es un cuestionario sencillo y fácil de cumplimentar diseñado específicamente para Podología, con resultados significativos de propiedades psicométricas que lo convierten en una herramienta fiable, válida y operativa, discrepando con otros autores en cuanto a estas propiedades ${ }^{16,17,18,19}$ y con una estructura de varios factores que evalúan las dimensiones acordadas de calidad asistencial.

Si consideramos que la factorización por sí misma es indicativa de Fiabilidad no sería necesario calcular el índice de alpha de Cronbach ${ }^{6,8}$. Nuestros resultados indican una consistencia interna alta, pero en ocasiones, debido a la heterogeneidad del constructo evaluado es conveniente priorizar la validez de contenido frente a la consistencia interna, si bien compartimos que esta validez depende en gran medida del grado de revisión de la literatura ${ }^{12}$. La validez de

Tabla 2. Análisis factorial. Varianza total

\begin{tabular}{cccr} 
Componente & Total & \% de la varianza & \% acumulado \\
\hline 1 & 8,796 & 38,244 & 38,244 \\
2 & 1,844 & 8,017 & 46,261 \\
3 & 1,557 & 6,770 & 53,031 \\
4 & 1,354 & 5,888 & 58,920 \\
5 & 1,123 & 4,884 & $\mathbf{6 3 , 8 0 4}$
\end{tabular}


criterio relaciona la puntuación de cada sujeto con un determinado "Gold Standard", criterios predictivos externos o de buena práctica ${ }^{5}$, proporcionando mayor validez. Se valoró con los ítems 25, 26 y 27.

Un requisito indispensable para la realización del análisis factorial es que la matriz de correlaciones inicial permita localizar agrupamientos de ítems. Primero, hay que calcular dos estimadores (test de esfericidad de Bartlett fue signi- ficativo y el índice de KMO mayor de 0,7) que aseguren que la matriz es adecuada ${ }^{20}$. Se realizó un análisis de la dimensionalidad comprobando que el cuestionario mide distintos aspectos de un constructo. Se identificó cinco dimensiones que explicaban una proporción importante de la varianza del modelo, determinando el número de factores con los ítems que contendrá cada factor y el grado de participación de cada variable en los factores.

Tabla 3. Rotación ortogonal de la matriz. Método varimax tras 8 rotaciones.

\begin{tabular}{|c|c|c|c|c|c|}
\hline \multicolumn{6}{|c|}{ Componentes } \\
\hline Item & 1 & 2 & 3 & 4 & 5 \\
\hline 22 & ,738 & , 159 &, 167 &, 170 &, 063 \\
\hline 18 & ,708 & 210 &, 002 & ,371 &, 116 \\
\hline 19 & 669 &, 356 & 031 & ,207 &, 085 \\
\hline 21 &, 591 &, 126 &, 321 & ,326 &, 168 \\
\hline 23 &, 560 & 219 & ,412 & ,023 &, 124 \\
\hline 24 &, 556 & ,201 & 036 & 081 &, 460 \\
\hline 16 &, 530 &, 416 &, 513 & ,032 &, 151 \\
\hline 10 & ,510 & ,351 & ,333 & ,324 & ,320 \\
\hline 15 & ,236 &, 846 & , 169 & ,018 & ,161 \\
\hline 13 & 290 &, 807 & 158 & ,241 & 020 \\
\hline 12 &, 024 & ,689 & ,143 & ,194 & ,136 \\
\hline 14 & ,214 & ,683 & ,270 & ,295 & ,009 \\
\hline 17 &, 455 &, 538 &, 513 & ,046 &, 116 \\
\hline 8 &, 083 & ,238 & ,733 & 064 &, 027 \\
\hline 9 & ,077 & ,186 & ,726 & , 145 &, 120 \\
\hline 20 & ,456 &, 056 &, 573 & ,269 &, 070 \\
\hline 1 & 145 & ,166 &, 553 & ,358 & ,358 \\
\hline 2 & 061 &, 244 &, 502 & ,349 & ,407 \\
\hline 11 &, 438 &, 257 & ,443 & ,036 &, 265 \\
\hline 6 & ,191 &, 242 & 039 & ,759 &, 112 \\
\hline 7 &, 159 &, 227 & ,302 &, 545 & 038 \\
\hline 5 & 258 & ,085 &, 383 & 477 &, 102 \\
\hline 3 & ,134 &, 021 & ,008 & ,154 & ,813 \\
\hline
\end{tabular}

Tabla 4. Clasificación de factores.

\begin{tabular}{lll} 
Factor & Denominación & Ítems \\
\hline 1 & Aspectos clínicos & $10,16,18,19,21,22,23,24$ \\
2 & Información & $12,13,14,15,17$ \\
3 & Administración e instalaciones & $1,2,8,9,11,20$ \\
4 & Tiempos y esperas & $5,6,7$ \\
5 & Accesos & 3
\end{tabular}


La comparación de los resultados estuvo dificultada por la inexistencia de trabajos similares en Podología. Sí son numerosos los cuestionarios diseñados para ámbito hospitalario y Atención Primaria ${ }^{5,6,8,10}$, algunos de ellos centrados en la satisfacción ${ }^{21}$. Destaca el estudio de Granado de la Orden, et al. con resultados parecidos $^{5}$ (análisis factorial con dos factores, $60 \%$ total de la varianza explicada, alpha de Cronbach de 0,8). Y González et al. con un análisis factorial ${ }^{22}$ del $50 \%$ de la varianza total explicada y alpha de Cronbach de 0,7. Las investigaciones desarrolladas en este ámbito de conocimiento han avanzado en el diseño de instrumentos de medida de la satisfacción para evaluar la calidad de los servicios, tanto en ámbito hospitalario ${ }^{23,24}$ como en Atención Primaria.

La satisfacción es una dimensión de la calidad de la atención podológica. Algunos autores consideran constructos diferentes los conceptos de calidad de servicio y satisfacción, los evalúan de forma separada ${ }^{25}$ y en ocasiones somos los profesionales los que no diferenciamos estos conceptos ${ }^{26}$. Una manera de valorar la calidad es relacionándola con la satisfacción de los usuarios ${ }^{27}$, que requiere un instrumento preciso para valorarla ${ }^{28}$. Por tanto, la satisfacción debe ser un objetivo irrenunciable para cualquier responsable de los servicios sanitarios y ha de ser vista como una medida más del resultado de sus intervenciones ${ }^{8,23}$.

La Podología defiende la importancia de los usuarios en el proceso de salud, su satisfacción es esencial para atraer y mantener a los usuarios en un ambiente sanitario competitivo ${ }^{26}$. Siendo probable que un paciente satisfecho utilice nuevamente los mismos servicios de sa$\mathrm{lud}^{18}$. El ámbito empresarial privado, contempla la satisfacción del usuario para conseguir la fidelidad, generar confianza y atraer a potenciales futuros clientes.

Los cuestionarios son usados con mayor frecuencia tanto en el ámbito asistencial como en la investigación, mediante su diseño (for- mato $^{29}$, extensión, etc.) podemos mejorar la validez y controlar posibles sesgos ${ }^{14}$. Este es un cuestionario validado con criterios de rigor metodológico para uso en ámbito específico de Podología.

Las categorías de respuesta son cinco, para no limitar las opciones y conseguir una distribución normal de respuesta. Siendo más simple que la testada de 1 a 10 y cualitativamente se comprende ${ }^{30}$. El ítem 4 es un ítem problemático, no tiene capacidad discriminante y se eliminó siguiendo recomendaciones ${ }^{31}$, pero tiene gran importancia y relevancia social.

En una siguiente etapa se pretende realizar un cuestionario con metodología que combine las técnicas cualitativas y cuantitativas ${ }^{16,17,18,19,26}$ que nos permita extraer información relevante coherente con los resultados descriptivos. Las investigaciones sobre los temas de calidad en Podología se reducen a los trabajos de Juárez ${ }^{32}$ sobre la influencia de la satisfacción en el tratamiento podológico y el tipo de financiación del mismo. Mahillo evaluó la calidad de las Historias clínicas ${ }^{33}$. Macran et al. que analizó el binomio pie-salud ${ }^{34}$ con un cuestionario de calidad de vida con seis dimensiones.

La elaboración de este cuestionario requiere de conocimientos de evaluación de gestión y calidad sanitaria. Pretende ser un instrumento eficaz, con fiabilidad y validez, para conocer la percepción de calidad asistencial, y que lo convierten en una herramienta útil para favorecer la gestión de los servicios de Podología ${ }^{35}$. Su uso periódico permitirá conocer debilidades y fortalezas existentes en cuanto a la percepción de calidad asistencial.

\section{AGRADECIMIENTOS}

A todo el personal del Departamento de Podología y del Área Clínica de Podología de la Universidad de Sevilla. A todos los usuarios del centro que son los que han inspirado y han contribuido en la realización de este proyecto.

\section{BIBLIOGRAFÍA}

1. Corbella A, Maturana S. Papel del ciudadano en los servicios de salud. Posibilidades y limitaciones. Rev Calid Asist.2000;15:357-362.

2. Varo J. La calidad de la atención médica. Med Clin.1995;104:538-540. 
3. Díaz R. Satisfacción del paciente: principal motor y centro de los servicios sanitarios. Rev Calid Asist.2002;17:9-22.

4. Villegas MM, Rosa IM. La calidad asistencial: concepto y medida. Rev Dirección, Organización y Administración Empresas.2003;29:50-58.

5. Granado de la Orden S, Rodríguez C, Olmedo C, et al. Diseño y validación de un cuestionario para evaluar la satisfacción de los pacientes atendidos en las consultas externas de un hospital de Madrid en 2.006. Rev Esp Salud Pública.2007;81:637-645.

6. González-Valentín MA, Padín S, Ramón E. Satisfacción del paciente con la atención de Enfermería. Enf Clínic.2005;15:147-155.

7. Duque EJ. Revisión de los modelos de evaluación de la calidad de servicio. Rev Innovar.2005;25:64-80.

8. Mira JJ, Buil JA, Aranaz JM, et al. ¿Qué opinan los pacientes de los hospitales públicos?: Análisis de los niveles de calidad percibida en cinco hospitales. Gac sanit.2000;14:291-293.

9. Thompson A, Suñol R. Las expectativas como factores en la satisfacción de los pacientes: conceptos, teorías y pruebas. Rev Calid Asist.1996;11:74-86.

10. Mira JJ, Aranaz JM, Buil JA, et al. SERVQHOS: un cuestionario para evaluar la calidad percibida de la asistencia hospitalaria. Med Prevent.1998;4:12-18.

11. Cronin JJ, Taylor SA. Servperf versus servqual. Reconciling performance-based and perceptionsminus-expectations measurement of service quality. J Marketing.1994;58:125-131.

12. Aranaz JM, Mira JJ. La satisfacción del paciente como una medida del resultado de la atención sanitaria. Med Clin.2000;114 (Supl 3):26-33.

13. Cárcamo CR. Calidad percibida: ¿Ilusión o percepción? Rev Calid Asist.2011; 26:184-187.

14. Martín MC. Diseño y validación de cuestionarios. Matronas profesión.2004; 17:23-29.

15. Carrillat FA, Jaramillo F, Mulki JP. The validity of the Servqual and Servperf scales: A meta-analytic view of 17 years of research across five continents. Intern J Serv Indust Manag.2007;18:472-490.

16. Caminnal J. La medida de la satisfacción: un instrumento de participación de la población en la mejora de la calidad de los servicios sanitarios. Rev Calid Asist. 2001;16:276-279.

17. Under L. Patient satisfaction measurement: current issues and implications. Outcomes Manag. 2002;6:125-131.

18. Otani K, Kurz RS, Harris L, et al. Managing Primary care using patient satisfaction measures. J Healthcare Manag.2005;50:311-324.

19. Hidalgo JR. La calidad en la provisión de la sanidad. Derechos de los pacientes como parámetro de calidad del servicio de salud. Rev Adm Sanit.2005;3:447-453.

20. Labarere J, Francois P, Anquier P, et al. Development of a french impatient satisfaction questionnaire. Intern J Qual Health Care.2001; 13:99-108.

21. Morales JM, Bonill C, Celdrán M, et al. Diseño y validación de instrumento de evaluación de la satisfacción con los servicios de atención domiciliaria: SATISFAD. Gac Sanit.2007;21:106-113.

22. González N, Quintana JM, Bilbao A, et al. Satisfacción de los usuarios de cuatro hospitales del Servicio Vasco de Salud. Gac Sanit.2008;22:210-7.

23. Mira JJ, Buil JA, Rodríguez J, et al. Calidad percibida del cuidado hospitalario. Gacet Sanit.1997;11:176-89.

24. Más A. Elaboración y validación de una encuesta de calidad percibida en pacientes hospitalizados. Rev Calid Asist.2001;16:501-510.

25. Thiedke CC. What do we really know about patient satisfaction? Fam Pract Manag.2007;14:33-36.

26. Sitzia J. How valid and reliable are patient satisfaction data? An analysis of 195 studies. Intern J Qual Health Care.1999;11:319-328.

27. Hendriks AA, Vrielink MR, Smets EA, et al. Improving the assessment of in patient's satisfaction with hospital care. Med Care.2001;39:270-283.

28. Argibay JC. Técnicas Psicométricas. Cuestiones de validez y confiabilidad. En: Subjetividad y Procesos cognitivos.2006;8:15-33.

29. Varela J, Rial A, García E. Presentación de una escala de satisfacción con los servicios sanitarios de Atención Primaria. Rev Psicothema.2003;15:656-661.

30. Collins $\mathrm{K}, \mathrm{O}^{\prime} \mathrm{C}$ athain A. The continuum of patient satisfaction from satisfied to very satisfied. Soc Sci Med.2003;57:2465-0.

31. Carretero-Dios H, Pérez C. Standards for the development and review of instrumental studies: Considerations absolute test selection in psychological research. Intern J Clin Health Pychology.2007;3:863-882. 
32. Juárez JM. Gratuidad versus equidad en la asistencia de pacientes en Podología [tesis doctoral]. Sevi1la: Departamento de Podología. Universidad de Sevilla; 2007.

33. Mahillo R. Evaluación de la calidad de las Historias clínicas en el Área Clínica de Podología de la Universidad de Sevilla. [tesis doctoral]. Sevilla: Departamento de Podología. Universidad de Sevilla; 2011.

34. Macran S, Collingwood J, Kind P, et al. Evaluating podiatry services: Testing a treatment specific measure of health status. Qual Life Res.2003;12:177-188.

35. Coheña M. Evaluación de la percepción de la calidad asistencial en el Área Clínica de Podología de la Universidad de Sevilla. [tesis doctoral]. Sevilla: Departamento de Podología. Universidad de Sevilla; 2012. 